\title{
Reservoir Potentials of the Cretaceous Gombe Sandstone, Gongola Basin, Upper Benue Trough, North Eastern Nigeria
}

\author{
B. Shettima ${ }^{1}$ I.Y. Buba, ${ }^{2}$ M. W. Sidi, ${ }^{1}$ Y. Abdulganiyu ${ }^{3}$, H. Hamidu, ${ }^{4}$ \\ U.Abubakar ${ }^{5}$ \\ ${ }^{1}$ Department of Geology University of Maiduguri, Borno State, Nigeria \\ ${ }^{2}$ Ministry of Solid Minerals, Gombe State Government, Nigeria \\ ${ }^{3}$ Department of Geology Federal University Birnin kebbi, Kebbi State, Nigeria. \\ ${ }^{4}$ Department of Geology Usmanu Danfodiyo University, Sokoto, Nigeria. \\ ${ }^{5}$ Department of Geology Gombe State University, Gombe, Nigeria.
}

\begin{abstract}
The Campano - Maastrichtian Gombe Sandstone of the Gongola Basin of the Upper Benue Trough is composed of arenaceous lithology that is laterally extensive and has reached a thickness of over $300 \mathrm{~m}$. The petrophysical evaluation of the formation on the basis of bivariate plots, petrographic analysis and falling head permeability indicated average porosity values of $15 \%, 33 \%$ and $23 \%$ respectively. Average permeability values of $5.2 \mathrm{D}$ and $100 \mathrm{md}$ were also obtained from bivariate plots and falling permeameter analysis respectively. These values are comparable to the reservoir rocks of proven petroleum basin of the North Sea and Niger Delta. Therefore, the Gombe Sandstone can serve as a potential reservoir rock in the Gongola Basin.
\end{abstract}

Keywords: Reservoir rock, porosity, permeability, bivariate plots, Gombe Sandstone, Gongola Basin

\section{Introduction}

Reservoir rock's ability to host and produce hydrocarbon is largely dependent on the degree of reservoir connectivity and the porosity/permeability characteristic of the reservoir unit acquired during sediment deposition and subsequent deposition. The Campano - Maastrichtian Gombe Sandstone of the Gongola Basin of the Upper Benue Trough is dominantly composed of arenaceous sediment which can possibly serve as a reservoir rock. Earlier sedimentological studies indicated that this formation is composed of quartz-arenite to arkosic sands which are mature to submature formed in a deltaic environment with thickness of over $300 \mathrm{~m}$ [1]. These coupled with relative position of the formation in the stratigraphy of Gongola Basin may suggest that the formation can serve as a potential reservoir rock in the basin. Therefore, the present research is aimed at evaluating the reservoir potential of the Gombe Sandstone.

\section{Stratigraphic Setting}

The Benue Trough is a major NE-SW trending rift basin of $50-150 \mathrm{~km}$ width and over $1000 \mathrm{~km}$ length. It is geographically sub-divided into Lower, Middle and Upper portion"Fig.1"[2]. The Upper Benue Trough is Y shaped made up of three arms, namely: the $\mathrm{E}-\mathrm{W}$ trending Yola Arm, N-S trending Gongola Arm or Gongola Basin and the NE - SW trending main arm (Muri - Lau Basin) (Dike, 2002) "Fig. 2".

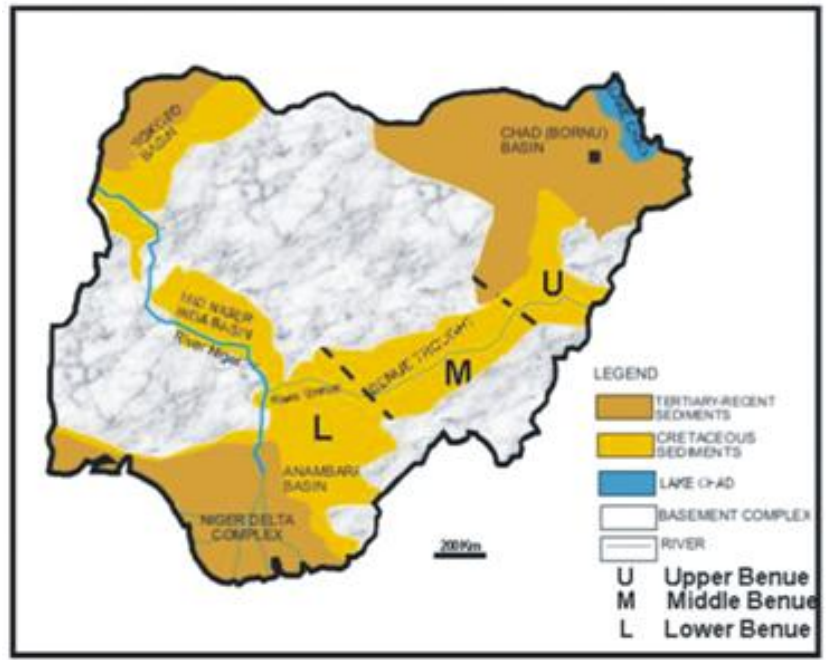

Figure 1: Geological Map of Nigeria showing the Benue Trough 


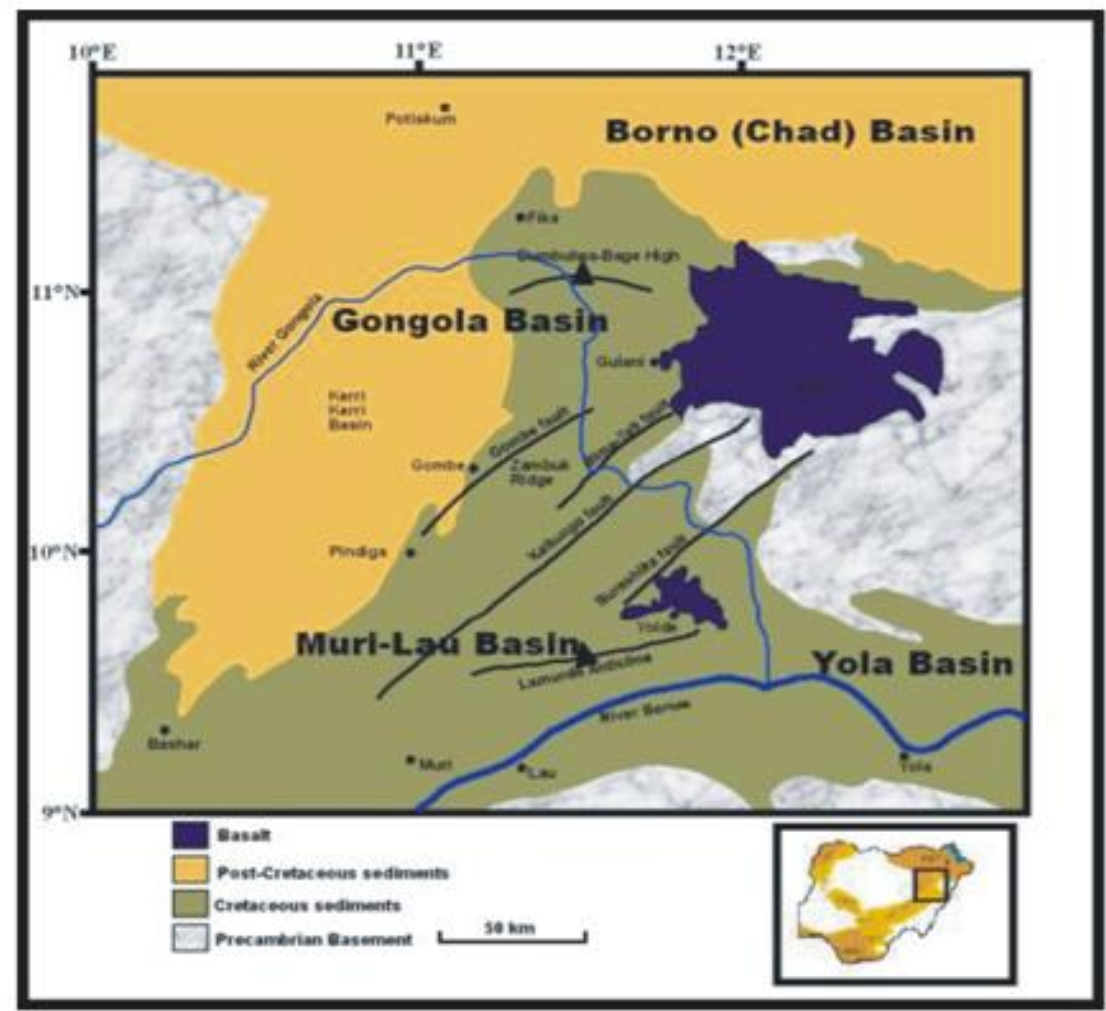

Figure 2: Geological Map of Upper Benue Trough (modified from Zaborski et al 1997)

In the Gongola Arm, the Aptian-Albian Bima Sandstone, a continental formation represents the basal part of the sedimentary succession. It unconformably overlies the Precambrian Basement Complex and consists of three siliciclastic members: the lower Bima (B1), middle Bima (B2) and the upper Bima (B3). Its lithology and depositional environments have been discussed by [3]'Fig. 3".

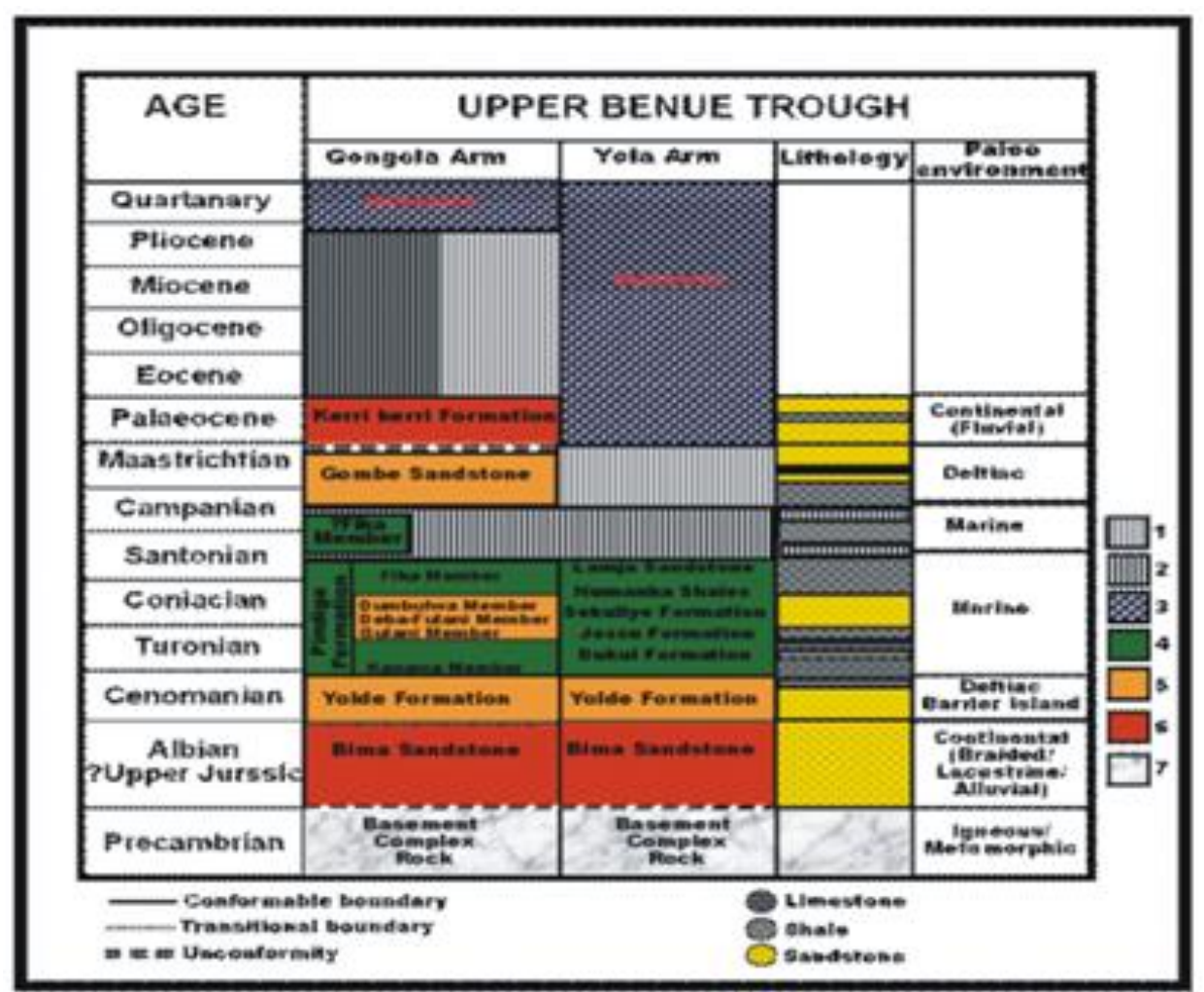

Figure3: Diagram showing the stratigraphy of the Upper Benue Trough (Obaje et al, 1999) 
The Yolde Formation lies conformably on the Bima Sandstone. It is Cenomanian in age [4] represents the beginning of marine incursion into the Gongola Arm. The Yolde Formation was deposited in a barrier island, deltaic settings [5] [6]. The Turonian-Campanian Pindiga Formation conformably overlies the Yolde Formation[7][8], [8] subdivided the Pindiga Formation into five lithostratigraphic members: the Kanawa Member which is the basal member comprises of limestone and shale intercalations, the Gulani Member, the Deban-Fulani Member, the Dumbulwa Member and the Fika Member which is the top-most member consisting of shale and very few limestones. The Gulani, Deba-fulani and the Dumbulwa members are lateral equivalents occurring in the middle part of the Pindiga Formation. They are deposited during the middle Turonian regional regressive episode that occurred in the Benue Trough [8].

The estuarine/deltaic Gombe Sandstone of Maastrichtian age [9] overlies the Pindiga Formation and it represents the youngest Cretaceous sediment in the Gongola Arm. The Paleocene Kerri-Kerri Formation unconformably overlies the Gombe Sandstone and represents the only record of Tertiary sedimentation in the Gongola Arm [10] [11].

\section{Methodology}

Twenty five samples of the Gombe Sandstone were collected around Gombe and environs where its thickest section occurs 'Fig. 4. These samples were collected from five outcrop sections with careful attention as to avoid weathered horizons 'Figs . 5, 6, 7, 8 and 9"Granulometric analysis was carried out by the conventional method and about $200 \mathrm{~g}$ of each sample was sieved for about 30 minutes in a Ro-Tap shaker. The graphical parameters of graphic mean, standard deviation, skewness and kurtosis were determined using the formula of Folk and Ward [12]. Petrographic analysis was also carried out on 30 samples using Ziess petrographical microscope to determine sorting, intergranular relationships, porosity and diagensis. Falling head permeameter test was likewise conducted on some few core samples according to the British standard (BS1377) procedure indicated by [13], in order to determine porosity and permeability for the Gombe Sandstone. Clay mineralogy of samples of the formation were analyzed using Shimadzu diffractometer (XRD-6000) coupled with discriminator, proportional counter, Co filter and $\mathrm{Cu}$ radiation at $40 \mathrm{kv}$ with scan range of $5-60^{\circ} 2 \Theta$.

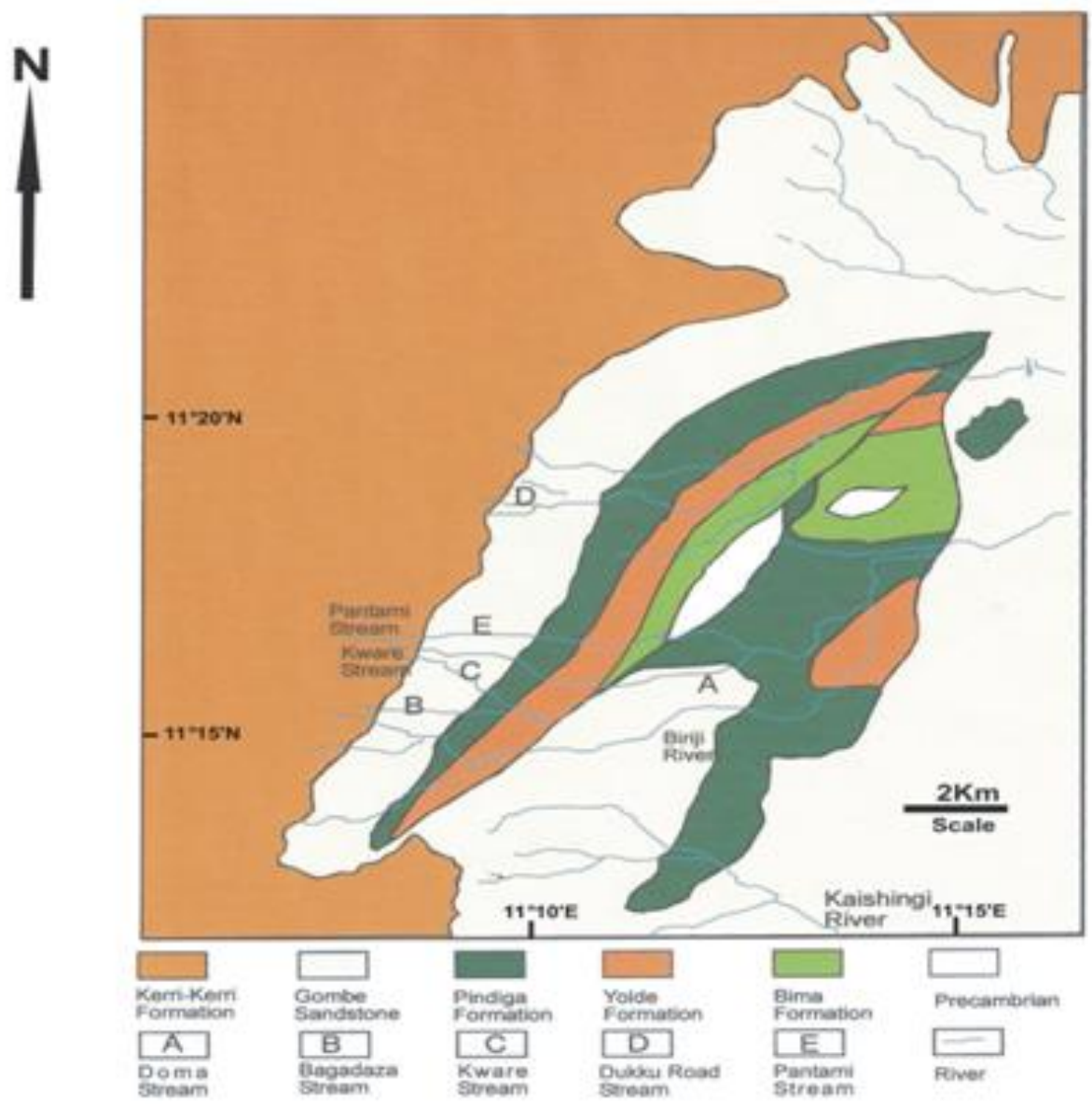

Figure 4: Geologic Map of Gombe and adjacent areas showing the location of section studied (modified from Zaborski et al, 1997) 


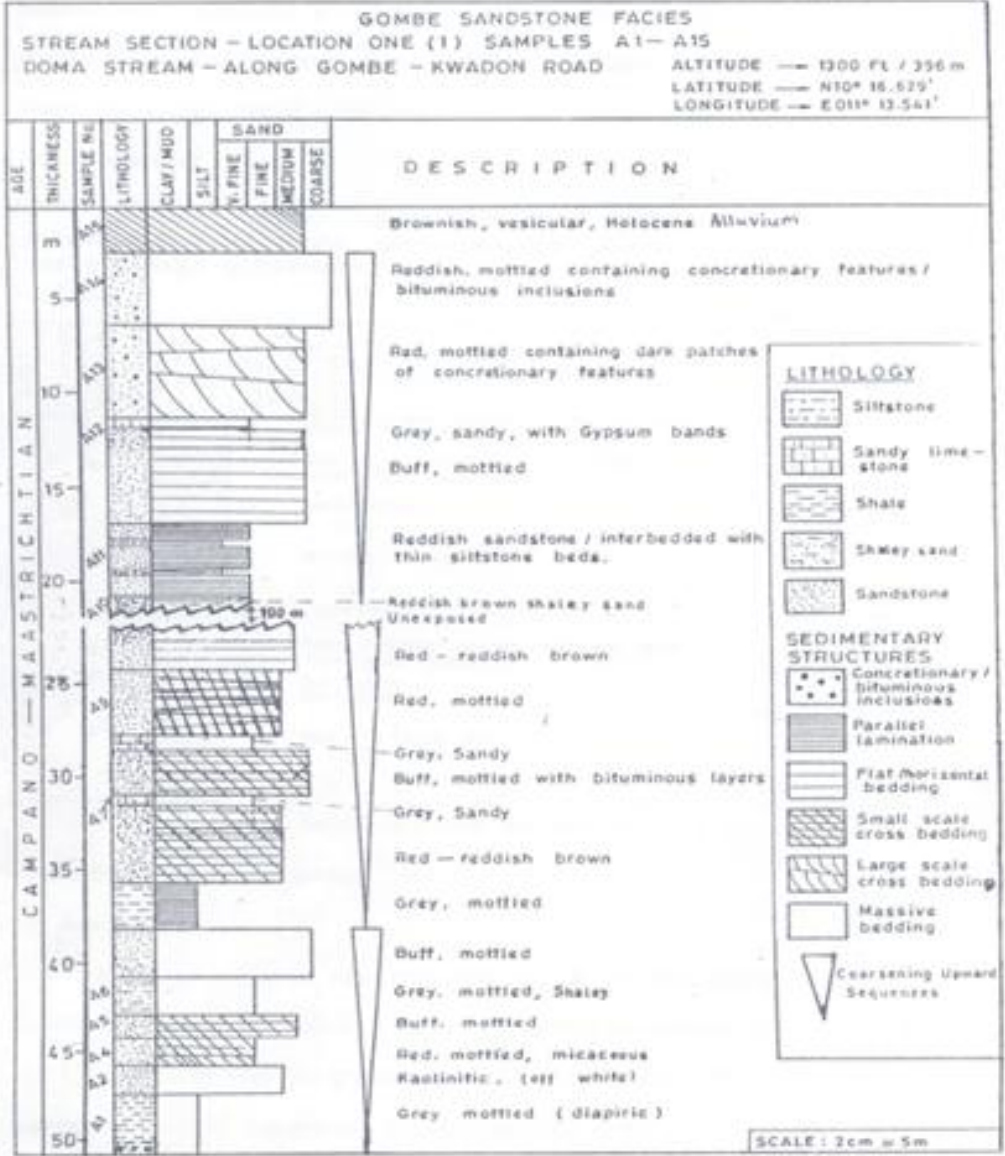

Figure 5: Section of Gombe sandstone at Doma stream

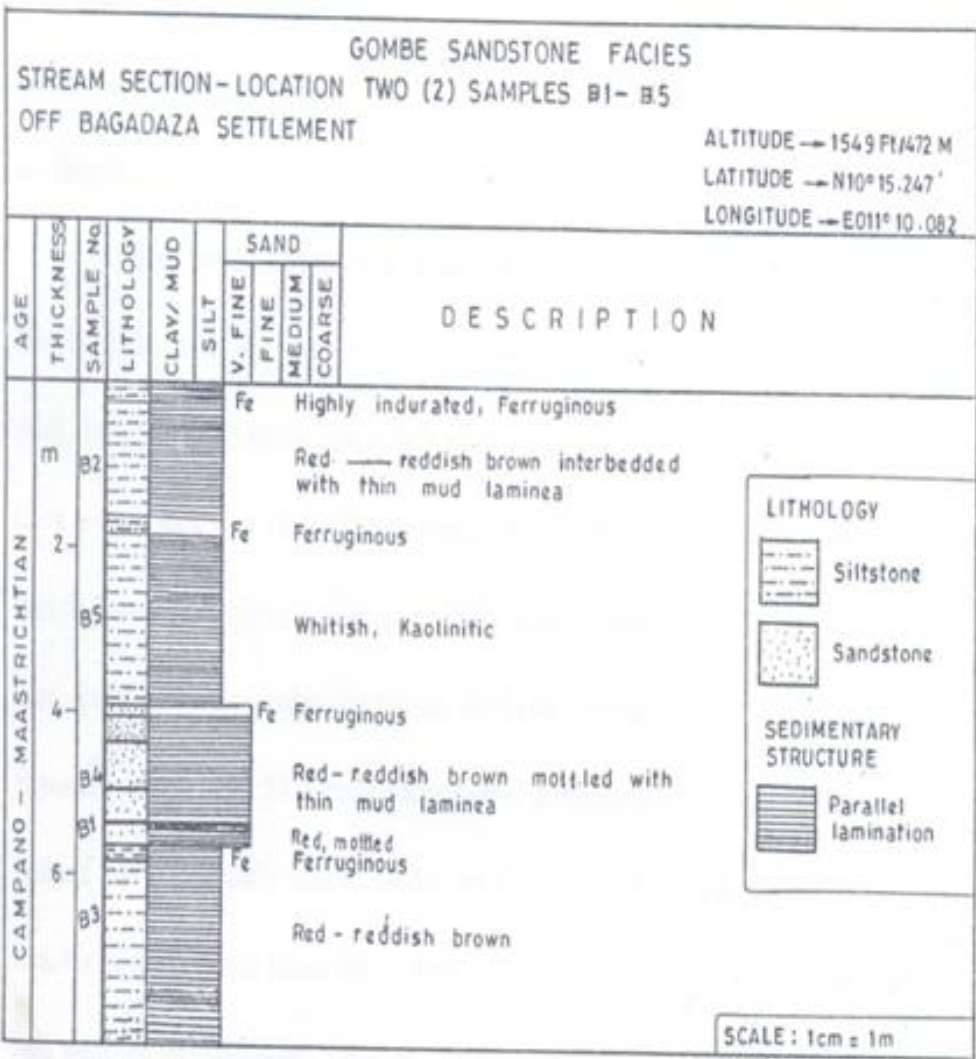

Figure 6: Section of Gombe sandstone at Bagadaza stream 


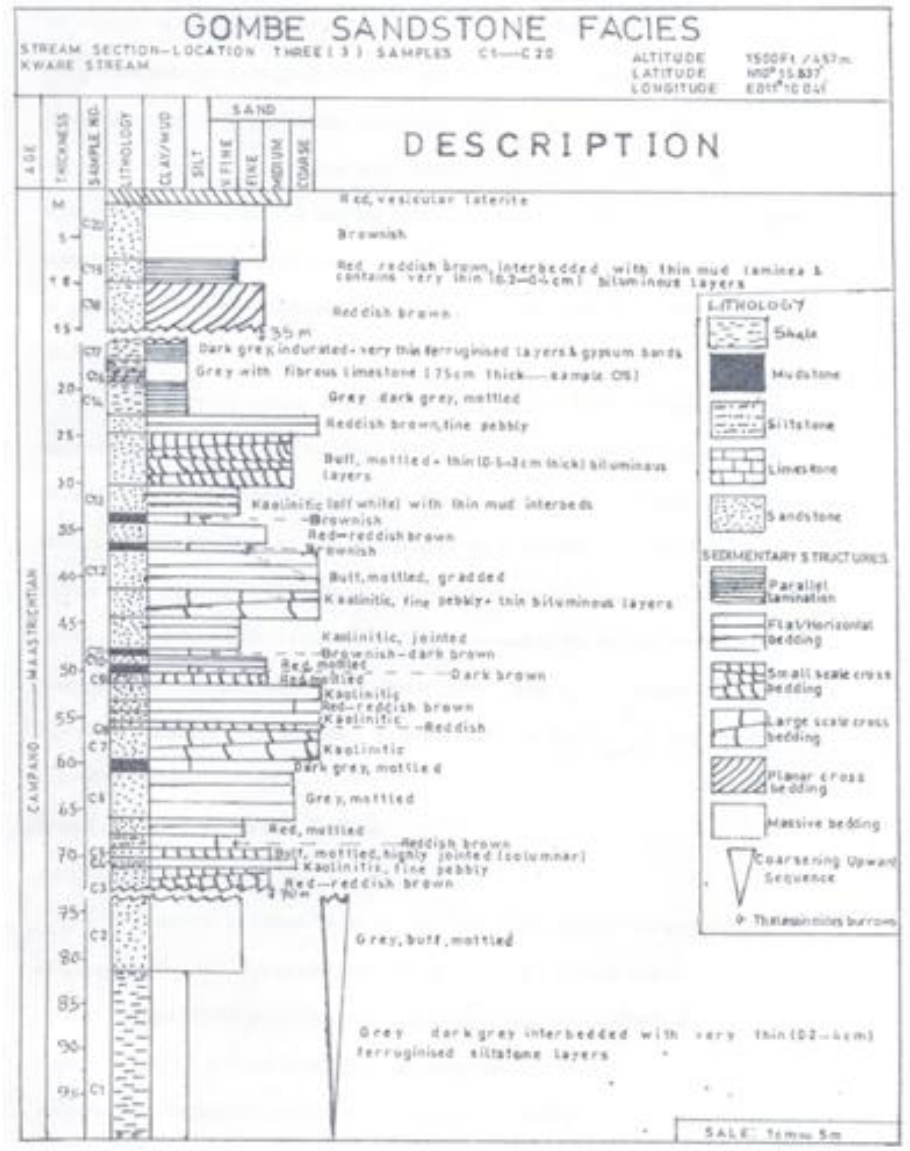

Figure 7: Section of Gombe sandstone at Kware stream

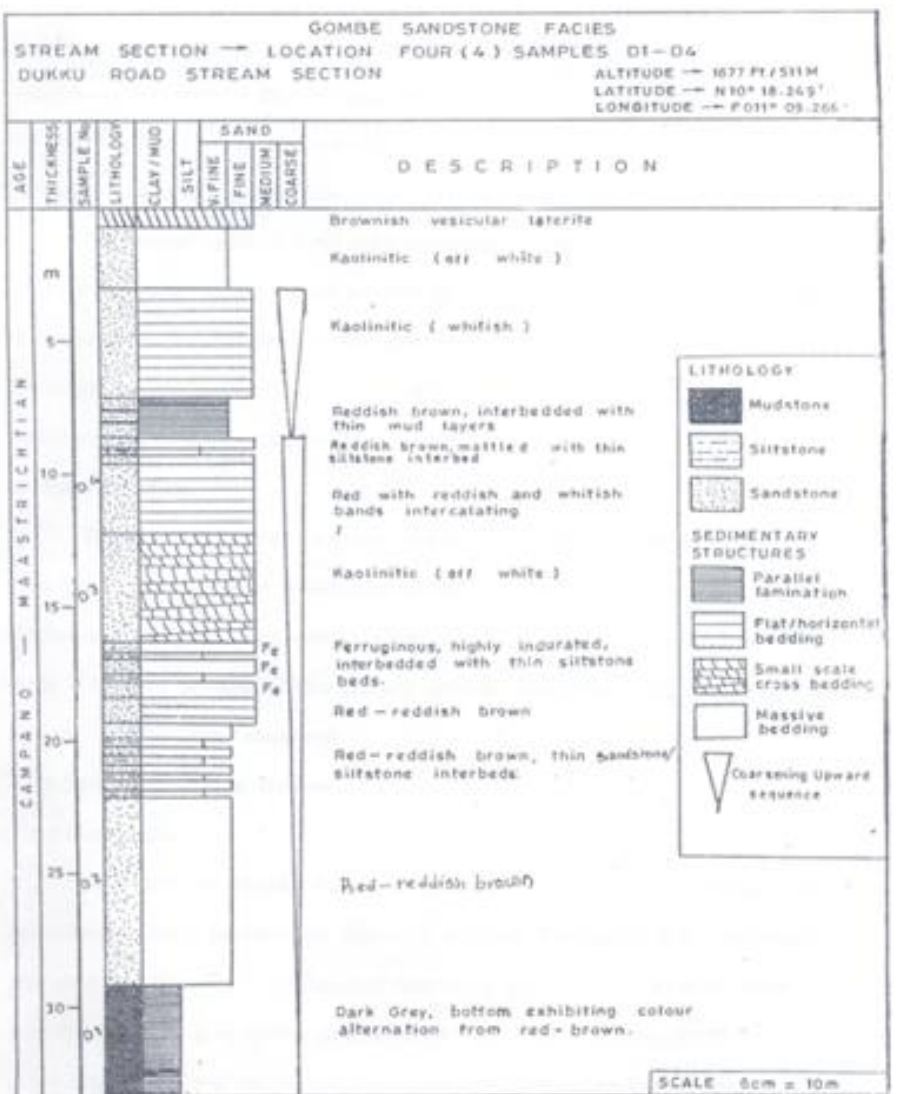

Figure 8: Section of Gombe Sandstone along Dukku road 


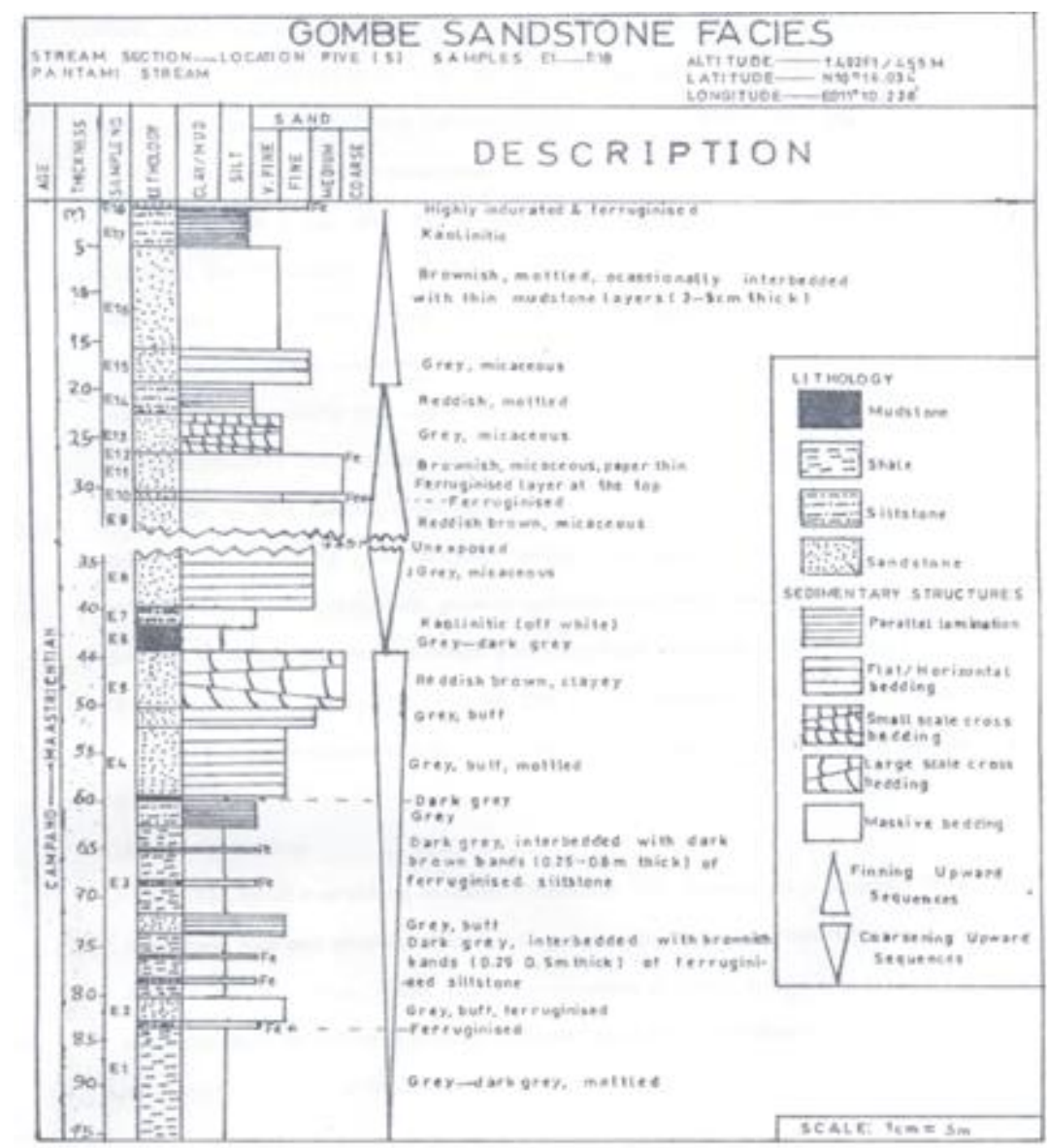

Figure 9: Section of Gombe Sandstone at Pantami

\subsection{Univariate grain size parameters:}

\section{Results}

The graphic mean size for the various samples"Table 1" range from $1.24 \varnothing-3.36 \emptyset$ i.e. (medium to very fine-grained sands ) and the fluctuation of the values may reflect change in the strength of the deposition medium. The values of standard deviation'Table 1"tend to show that the samples ranged from well sorted $(0.48 \varnothing)$ to poorly sorted $(2.09 \varnothing)$ with an average of $(1.17 \varnothing)$ which implies that the whole formation is poorly sorted.

The samples analysed have skewness values ranging from $-0.12 \varnothing$ to $0.44 \varnothing$ i.e. from negatively skewed to very positively skewed respectively. However, positively skewed values predominate"Table 1"with an average of $0.25 \varnothing$, and this may be due to the fact that much of the silt and clay were not removed by current , though the clay may be secondary. The values of kurtosis"Table 1" for the various samples range from $0.62 \varnothing$ to $2.39 \varnothing$ (very platykurtic to very leptokurtic), with an average of $1.00 \varnothing$ (mesokurtic).

\subsection{Petrographic study}

Twenty samples ranging from fine to coarse grained sandstone were thin sectioned and subjected to petrographic studies. The sandstones consist mainly of quartz, feldspars and mica as framework elements with clay matrix and cement. The framework composition of these sandstones is varied and is presented in" Table 2 ". The texture of these sandstones tends to show that sorting ranges from poorly to well sorted $\quad(1.0-0.4)$ but moderate sorting predominates'Table 2". The grain shape ranges from subangular to well rounded $(0.2-0.5)$ with sub-angular dominating, and the sphericity varies from low-high $(0.3-0.9)^{\prime}$ Figs. $11-16$.

Quartz comprises an average of $73 \%$ of the framework grain of the sandstones. Monocrystalline quartz is dominant, while polycrystalline quartz occurs in very few samples. Most of the quartz grains are characterized by various features such as dust lines, and quartz overgrowth.The feldspars generally range from $4-15 \%$ and Potassium feldspars dominate, followed by plagioclase feldspar. Seriticization of the feldspar is quite a common feature in most of the studied samples. Muscovite makes up to $(1 \%)$ of the grains and opaque minerals range between $(1-2 \%)$ in the samples analysed.Using [14] sandstone classification, the Yolde Formation sandstone range from subarkose to quartzarenite, and their textural maturity based on [15] ranges from submature to mature.Petrographic evaluation of the porosity based on estimation shows that the Gombe Sandstone has porosity values ranging from $7-19 \%$ with an average of $19 \%$ "Table 2 ". 
Table 1 Showing univariate grain size parameters and porosity and permeability data

\begin{tabular}{|c|c|c|c|c|c|c|}
\hline $\begin{array}{l}\text { SAMPL } \\
\text { E No. }\end{array}$ & $\begin{array}{l}\text { MEAN } \\
\text { (Mz) } \Phi\end{array}$ & $\begin{array}{l}\text { STANDARD } \\
\text { DEVIATION } \\
\text { (SORTING) } \Phi\end{array}$ & $\begin{array}{l}\text { SKEWNESS } \\
\text { (Ski) } \Phi\end{array}$ & $\begin{array}{l}\text { KURTOSIS } \\
\text { (Kc) } \Phi\end{array}$ & $\begin{array}{l}\text { POROSIT } \\
\text { Y }(\%)\end{array}$ & $\begin{array}{l}\text { PERMEABI } \\
\text { LITY (D) }\end{array}$ \\
\hline A4 & $\begin{array}{l}3.11 \\
\text { Very fine } \\
\text { grained }\end{array}$ & $\begin{array}{l}0.86 \\
\text { Moderately } \\
\text { sorted }\end{array}$ & $\begin{array}{l}0.28 \\
\text { Positively } \\
\text { skewed }\end{array}$ & $\begin{array}{l}0.77 \\
\text { Platykurtic }\end{array}$ & 38.5 & 4.7 \\
\hline A5 & $\begin{array}{l}3.05 \\
\text { Very fine } \\
\text { grained }\end{array}$ & $\begin{array}{l}0.89 \\
\text { Moderately } \\
\text { Sorted }\end{array}$ & $\begin{array}{l}0.23 \\
\text { Positively } \\
\text { skewed }\end{array}$ & $\begin{array}{l}0.94 \\
\text { Mesokurtic }\end{array}$ & 40.2 & 4.2 \\
\hline A9 & $\begin{array}{l}3.02 \\
\text { Very fine } \\
\text { grained }\end{array}$ & $\begin{array}{l}097 \\
\text { Moderately } \\
\text { Sorted } \\
\end{array}$ & $\begin{array}{l}0.15 \\
\text { Positively } \\
\text { skewed }\end{array}$ & $\begin{array}{l}0.70 \\
\text { Platykurtic }\end{array}$ & 38.5 & 5.2 \\
\hline A10 & $\begin{array}{l}3.31 \\
\text { Very fine } \\
\text { grained }\end{array}$ & $\begin{array}{l}0.77 \\
\text { Moderately } \\
\text { Sorted }\end{array}$ & $\begin{array}{l}0.18 \\
\text { Negatively } \\
\text { skewed }\end{array}$ & $\begin{array}{l}0.62 \\
\text { Platykurtic }\end{array}$ & 40 & 5 \\
\hline A13 & $\begin{array}{l}2.53 \\
\text { Fine grained }\end{array}$ & $\begin{array}{l}1.21 \\
\text { Poorly Sorted }\end{array}$ & $\begin{array}{l}0.36 \\
\text { Positively } \\
\text { skewed }\end{array}$ & $\begin{array}{l}0.98 \\
\text { Mesokurtic }\end{array}$ & 31 & 5 \\
\hline A14 & $\begin{array}{l}2.27 \\
\text { Fine grained }\end{array}$ & $\begin{array}{l}1.32 \\
\text { Poorly Sorted }\end{array}$ & $\begin{array}{l}0.41 \\
\text { Positively } \\
\text { skewed }\end{array}$ & $\begin{array}{l}1.14 \\
\text { Leptokurtic }\end{array}$ & 29 & 4 \\
\hline B1 & $\begin{array}{l}3,37 \\
\begin{array}{l}\text { Very fine } \\
\text { grained }\end{array}\end{array}$ & $\begin{array}{l}0.56 \\
\text { Moderately } \\
\text { sorted }\end{array}$ & $\begin{array}{l}0.00 \\
\text { Symmetrical }\end{array}$ & $\begin{array}{l}1.34 \\
\text { Leptokurtic }\end{array}$ & 41 & 7 \\
\hline B4 & $\begin{array}{l}3.36 \\
\begin{array}{l}\text { Very fine } \\
\text { grained }\end{array}\end{array}$ & $\begin{array}{l}0.48 \\
\text { Well sorted }\end{array}$ & $\begin{array}{l}0.11 \\
\text { Nearly } \\
\text { symmetrical }\end{array}$ & $\begin{array}{l}1.34 \\
\text { Leptokurtic }\end{array}$ & 41 & 6.5 \\
\hline C3 & $\begin{array}{l}2.38 \\
\text { Fine grained }\end{array}$ & $\begin{array}{l}1,44 \\
\text { Poorly sorted }\end{array}$ & $\begin{array}{l}0.12 \\
\text { Positively } \\
\text { skewed }\end{array}$ & $\begin{array}{l}0.74 \\
\text { Very } \\
\text { platykurtic }\end{array}$ & 28.5 & 3.5 \\
\hline $\mathrm{C} 4$ & $\begin{array}{l}1.79 \\
\text { Medium } \\
\text { grained }\end{array}$ & $\begin{array}{l}1.79 \\
\text { Poorly Sorted }\end{array}$ & $\begin{array}{l}0.27 \\
\text { Positively } \\
\text { skewed }\end{array}$ & $\begin{array}{l}0.88 \\
\text { Platykurtic }\end{array}$ & 25 & 0.2 \\
\hline C5 & $\begin{array}{l}2.06 \\
\text { Fine } \\
\text { grained }\end{array}$ & $\begin{array}{l}1.52 \\
\text { Poorly sorted }\end{array}$ & $\begin{array}{l}0.38 \\
\text { Positively } \\
\text { skewed }\end{array}$ & $\begin{array}{l}0.85 \\
\text { Platykurtic }\end{array}$ & 27.5 & 3 \\
\hline C6 & $\begin{array}{l}1.94 \\
\text { Fine grained }\end{array}$ & $\begin{array}{l}1.60 \\
\text { Poorly Sorted }\end{array}$ & $\begin{array}{l}0.39 \\
\text { Positively } \\
\text { skewed }\end{array}$ & $\begin{array}{l}0.88 \\
\text { Platykurtic }\end{array}$ & 27 & 1.5 \\
\hline C7 & $\begin{array}{l}1.77 \\
\text { Medium } \\
\text { grained }\end{array}$ & $\begin{array}{l}1.83 \\
\text { Poorly Sorted }\end{array}$ & $\begin{array}{l}0.32 \\
\text { Positively } \\
\text { skewed }\end{array}$ & $\begin{array}{l}0.82 \\
\text { Very } \\
\text { platykurtic }\end{array}$ & 26 & 0.1 \\
\hline C8 & $\begin{array}{l}1.64 \\
\text { Medium } \\
\text { grained }\end{array}$ & $\begin{array}{l}1.84 \\
\text { Poorly Sorted }\end{array}$ & $\begin{array}{l}0.44 \\
\text { Positively } \\
\text { skewed }\end{array}$ & $\begin{array}{l}1.26 \\
\text { Leptokurtic }\end{array}$ & 26 & 0.15 \\
\hline C9 & $\begin{array}{l}2.06 \\
\text { Fine grained }\end{array}$ & $\begin{array}{l}1.49 \\
\text { Poorly Sorted }\end{array}$ & $\begin{array}{l}0.35 \\
\text { Positively } \\
\text { skewed }\end{array}$ & $\begin{array}{l}0.93 \\
\text { Mesokurtic }\end{array}$ & 27 & 2.8 \\
\hline C12 & $\begin{array}{l}2.26 \\
\text { Fine grained }\end{array}$ & $\begin{array}{l}1.06 \\
\text { Poorly Sorted }\end{array}$ & $\begin{array}{l}0.09 \\
\text { Nearly } \\
\text { Symmetrical }\end{array}$ & $\begin{array}{l}1.19 \\
\text { Leptokurtic }\end{array}$ & 35.5 & 15 \\
\hline $\mathrm{C} 18$ & $\begin{array}{l}3.16 \\
\begin{array}{l}\text { Very fine } \\
\text { grained }\end{array}\end{array}$ & $\begin{array}{l}0.71 \\
\text { Moderately } \\
\text { Sorted }\end{array}$ & $\begin{array}{l}0.37 \\
\text { Positively } \\
\text { skewed }\end{array}$ & $\begin{array}{l}0.84 \\
\text { Platykurtic }\end{array}$ & 39 & 6 \\
\hline D2 & $\begin{array}{l}2.92 \\
\text { Fine grained }\end{array}$ & $\begin{array}{l}0.90 \\
\text { Moderately } \\
\text { Sorted } \\
\end{array}$ & $\begin{array}{l}0.02 \\
\text { Nearly } \\
\text { symmetrical }\end{array}$ & $\begin{array}{l}1.49 \\
\text { Leptokurtic }\end{array}$ & 37 & 5 \\
\hline D3 & $\begin{array}{l}2.76 \\
\text { Fine grained }\end{array}$ & $\begin{array}{l}0.83 \\
\text { Moderately } \\
\text { Sorted }\end{array}$ & $\begin{array}{l}-0.12 \\
\text { Negatively } \\
\text { skewed }\end{array}$ & $\begin{array}{l}2.39 \\
\text { Very } \\
\text { leptokurtic }\end{array}$ & 37 & 7.5 \\
\hline D4 & $\begin{array}{l}3.15 \\
\begin{array}{l}\text { Very fine } \\
\text { grained }\end{array}\end{array}$ & $\begin{array}{l}0.73 \\
\text { Moderately } \\
\text { sorted }\end{array}$ & $\begin{array}{l}0.44 \\
\text { Positively } \\
\text { skewed }\end{array}$ & $\begin{array}{l}1.03 \\
\text { Mesokurtic }\end{array}$ & 40.5 & 7 \\
\hline E5 & $\begin{array}{l}1.24 \\
\text { Medium } \\
\text { grained }\end{array}$ & $\begin{array}{l}2.09 \\
\text { Very poorly } \\
\text { Sorted }\end{array}$ & $\begin{array}{l}0.24 \\
\text { Positively } \\
\text { skewed }\end{array}$ & $\begin{array}{l}1.02 \\
\text { Mesokurtic }\end{array}$ & 25 & 0.1 \\
\hline E9 & $\begin{array}{l}2.26 \\
\text { Fine grained }\end{array}$ & $\begin{array}{l}1.14 \\
\text { Poorly Sorted }\end{array}$ & $\begin{array}{l}0.38 \\
\text { Positively } \\
\text { skewed }\end{array}$ & $\begin{array}{l}1.10 \\
\text { Mesokurtic }\end{array}$ & 27 & 12 \\
\hline E11 & $\begin{array}{l}1.73 \\
\text { Medium } \\
\text { grained }\end{array}$ & $\begin{array}{l}1.41 \\
\text { Poorly sorted }\end{array}$ & $\begin{array}{l}0.16 \\
\text { Positively } \\
\text { skewed }\end{array}$ & $\begin{array}{l}1.04 \\
\text { Mesokurtic }\end{array}$ & 31 & 13 \\
\hline
\end{tabular}


Reservoir Potentials of the Cretaceous Gombe Sandstone, Gongola Basin, Upper Benue Tough, North

\begin{tabular}{|l|l|l|l|l|l|l|}
\hline E13 & $\begin{array}{l}3.17 \\
\text { Very fine } \\
\text { grained }\end{array}$ & $\begin{array}{l}0.78 \\
\text { Moderately } \\
\text { Sorted }\end{array}$ & $\begin{array}{l}0.33 \\
\text { Positively } \\
\text { skewed }\end{array}$ & $\begin{array}{l}0.70 \\
\text { Platykurtic }\end{array}$ & 39 & 6 \\
\hline E15 & $\begin{array}{l}2.92 \\
\text { Fine grained }\end{array}$ & $\begin{array}{l}0.93 \\
\text { Moderately } \\
\text { Sorted }\end{array}$ & $\begin{array}{l}0.28 \\
\text { Positively } \\
\text { skewed }\end{array}$ & $\begin{array}{l}1.02 \\
\text { Mesokurtic }\end{array}$ & 37 & 5.5 \\
\hline Average & $\begin{array}{l}\mathbf{2 . 5 5} \\
\text { Fine } \\
\text { grained }\end{array}$ & $\begin{array}{l}\mathbf{1 . 1 7} \\
\text { Poorly sorted }\end{array}$ & $\begin{array}{l}\mathbf{0 . 2 5} \\
\text { Positively } \\
\text { skewed }\end{array}$ & $\begin{array}{l}\mathbf{1 . 0 0} \\
\text { Mesokurtic }\end{array}$ & $\mathbf{3 3 . 3 7}$ & $\mathbf{5 . 2 0}$ \\
\hline
\end{tabular}

II. Table 2 Showing the petrographic framework components and optical porosity of the Gombe Sandstone

\begin{tabular}{|c|c|c|c|c|c|c|c|c|c|c|c|}
\hline S/No & $\begin{array}{l}\text { SAMPLE } \\
\text { NO. }\end{array}$ & $\begin{array}{c}\text { SORTING } \\
1\end{array}$ & $\begin{array}{c}\text { ROUNDNESS } \\
2\end{array}$ & $\begin{array}{c}\text { SPHERICITY } \\
\mathbf{2}\end{array}$ & $\begin{array}{c}\text { GRAIN FABRIC } \\
3 \\
\end{array}$ & \multicolumn{6}{|c|}{$\begin{array}{c}\text { FRAME WORK COMPONENT } \\
\qquad 4\end{array}$} \\
\hline & & & & & & $\begin{array}{l}\text { Quartz } \\
(\%)\end{array}$ & $\begin{array}{c}\text { Feldspar } \\
(\%)\end{array}$ & $\begin{array}{c}\text { Mica } \\
(\%)\end{array}$ & $\begin{array}{c}\text { Opaque } \\
(\%)\end{array}$ & $\begin{array}{c}\text { Cement } \\
\text { Matrix (\%) }\end{array}$ & $\begin{array}{c}\text { Porosity } \\
(\%)\end{array}$ \\
\hline 1 & A4 & Moderately sorted $(0.4)$ & Subrounded $(0.4)$ & 0.9 (high) & Grain Supported & 72 & 8 & 1 & 1 & 4 & 18 \\
\hline 2 & A5 & Moderately sorted $(0.5)$ & Subangular $(0.3)$ & 0.4 (Low) & Grain Supported & 70 & 10 & 1 & 1 & 3 & 16 \\
\hline 3 & A9 & Moderately sorted $(0.5)$ & Subangular $(0.3)$ & 0.3 (low) & Grain Supported & 68 & 10 & 1 & 1 & 5 & 15 \\
\hline 4 & A10 & Moderately sorted $(0.5)$ & Subangular $(0.3)$ & 0.3 (low) & Grain Supported & 65 & 12 & 1 & 2 & 4 & 18 \\
\hline 5 & A13 & Poorly sorted (1.0) & Subangular (0.3) & 0.3 (low) & Grain Supported & 65 & 13 & 1 & 2 & 2 & 16 \\
\hline 6 & A14 & Poorly sorted $(1.0)$ & Subangular $(0.3)$ & 0.3 (low) & Grain Supported & 67 & 10 & - & 1 & 4 & 18 \\
\hline 7 & B1 & Moderately sorted (1.0) & Subangular (0.3) & 0.2 (low) & Grain Supported & 68 & 12 & 1 & 1 & 5 & 18 \\
\hline 8 & B4 & Well sorted $(0.3)$ & Subangular $(0.3)$ & 0.8 (high) & Grain Supported & 65 & 15 & - & 2 & 5 & 17 \\
\hline 9 & $\mathrm{C} 3$ & Poorly sorted (1.0) & Subrounded $(0.4)$ & 0.3 (low) & Grain Supported & 70 & 8 & - & 1 & 4 & 18 \\
\hline 10 & $\mathrm{C} 4$ & Poorly sorted $(1.0)$ & Subangular $(0.3)$ & 0.3 (low) & Grain Supported & 68 & 11 & 1 & 1 & 4 & 11 \\
\hline 11 & $\mathrm{C} 5$ & Poorly sorted (1.0) & Subangular $(0.3)$ & 0.3 (low) & Grain Supported & 65 & 15 & - & 2 & 3 & 14 \\
\hline 12 & C6 & Poorly sorted (1.0) & Subangular $(0.3)$ & 0.3 (Low) & Grain Supported & 70 & 8 & - & 1 & 4 & 12 \\
\hline 13 & C7 & Poorly sorted (1.0) & Subangular $(0.3)$ & 0.6 (high) & Grain Supported & 68 & 13 & 1 & 1 & 3 & 17 \\
\hline 14 & C8 & Poorly sorted $(1.0)$ & Subrounded $(0.4)$ & 0.9 (high) & Grain Supported & 72 & 8 & - & 1 & 4 & 16 \\
\hline 15 & $\mathrm{C} 9$ & Poorly sorted (1.0) & Subrounded $(0.4)$ & 0.9 (high) & Grain Supported & 70 & 10 & 1 & 2 & 4 & 15 \\
\hline 16 & C12 & Poorly sorted $(1.0)$ & Well rounded $(0.6)$ & 0.9 (high) & Grain Supported & 95 & 2 & - & 1 & 3 & 13 \\
\hline 17 & C18 & Well sorted $(0.4)$ & Rounded $(0.5)$ & 0.9 (high) & Grain Supported & 95 & 3 & - & 1 & 2 & 8 \\
\hline 18 & D2 & Well sorted $(0.4)$ & Well rounded $(0.6)$ & 0.9 (high) & Grain Supported & 96 & 2 & - & 1 & 2 & 15 \\
\hline 19 & D3 & Moderately sorted $(0.5)$ & Subrounded $(0.4)$ & 0.9 (high) & Grain Supported & 70 & 8 & 1 & 1 & 4 & 15 \\
\hline 20 & D4 & Moderately sorted $(0.5)$ & Subangular $(0.3)$ & 0.7 (high) & Grain Supported & 70 & 12 & 1 & 2 & 5 & 15 \\
\hline 21 & E5 & Poorly sorted $(1.0)$ & Subangular (0.3) & 0.8 (high) & Grain Supported & 72 & 10 & 1 & 1 & 3 & 14 \\
\hline 22 & E9 & Moderately sorted $(0.5)$ & Subangular (0.3) & 0.7 (high) & Grain Supported & 69 & 15 & - & 1 & 4 & 16 \\
\hline 23 & E11 & Moderately sorted $(0.5)$ & Subangular $(0.3)$ & 0.3 (low) & Grain Supported & 73 & 10 & - & 2 & 3 & 8 \\
\hline 24 & E13 & Well sorted $(0.4)$ & Rounded $(0.5)$ & 0.9 (high) & Grain Supported & 80 & 8 & 1 & 2 & 4 & 16 \\
\hline 25 & E15 & Moderately sorted $(0.5)$ & Subangular $(0.3)$ & 0.3 (low) & Grain Supported & 62 & 15 & 1 & 2 & 5 & 11 \\
\hline
\end{tabular}

1 - Estimates based on Folk (1972), 2 - Estimates based on Powers (1953), 3 - Estimates based on Swanson (1985), 4 - Estimates based on Terry and Chillinger (1955)

III. Table 2 Showing the petrographic framework components and optical porosity of the Gombe Sandstone

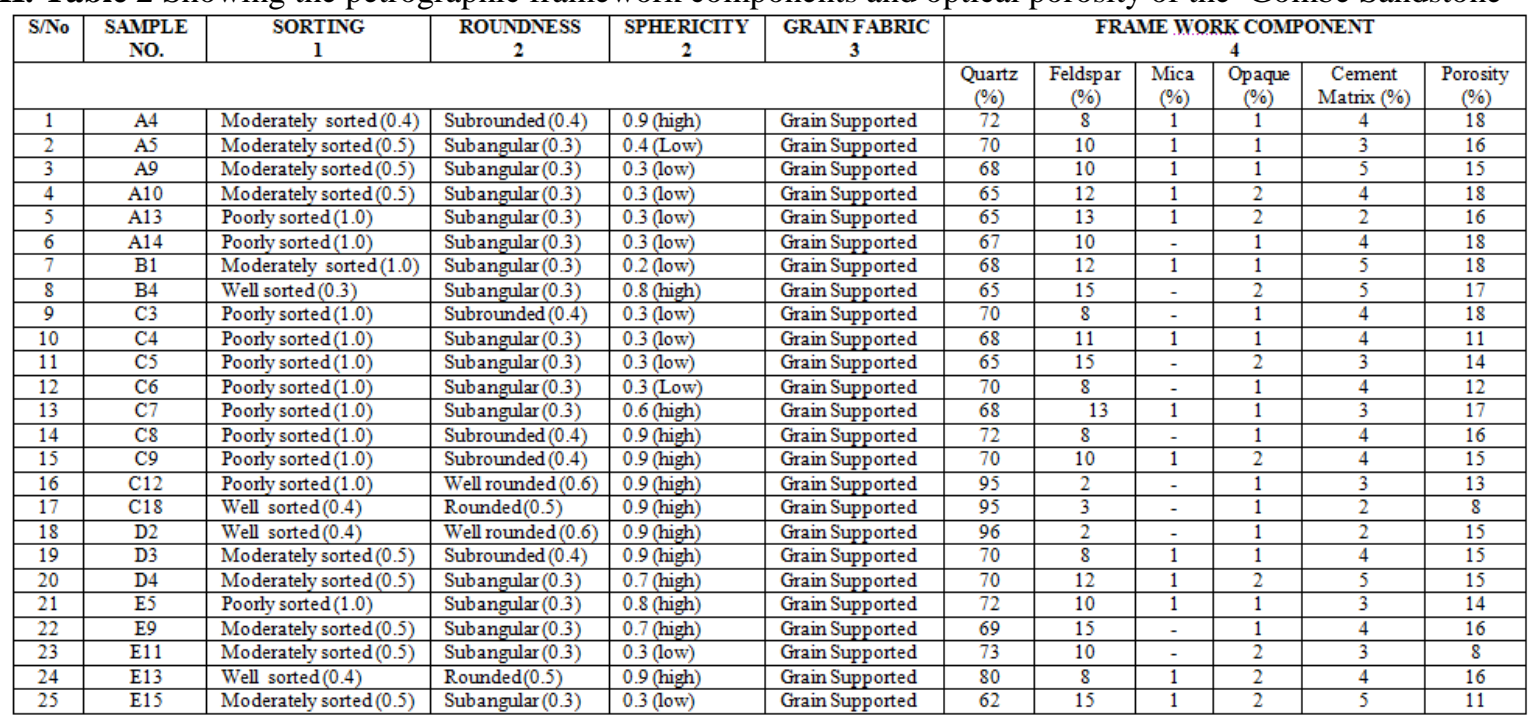

\section{1- Estimates based on Folk (1972), 2 - Estimates based on Powers (1953), 3 - Estimates based on}

\section{Bivariate plots}

The bivariate plots of grain size versus standard deviation [16] for evaluation of petrophysical properties was also employed in this studies to determine the porosity and permeability of the Gombe Sandstone. Twenty five (25) samples were plotted in the diagram'Fig .10', and the porosity ranged from $26 \%$ $42 \%$, with an average of $35 \%$. While the permeability varied from $0.5 \mathrm{D}-14 \mathrm{D}$ with an average of $6.5 \mathrm{D}$ 'Table 1 ". 


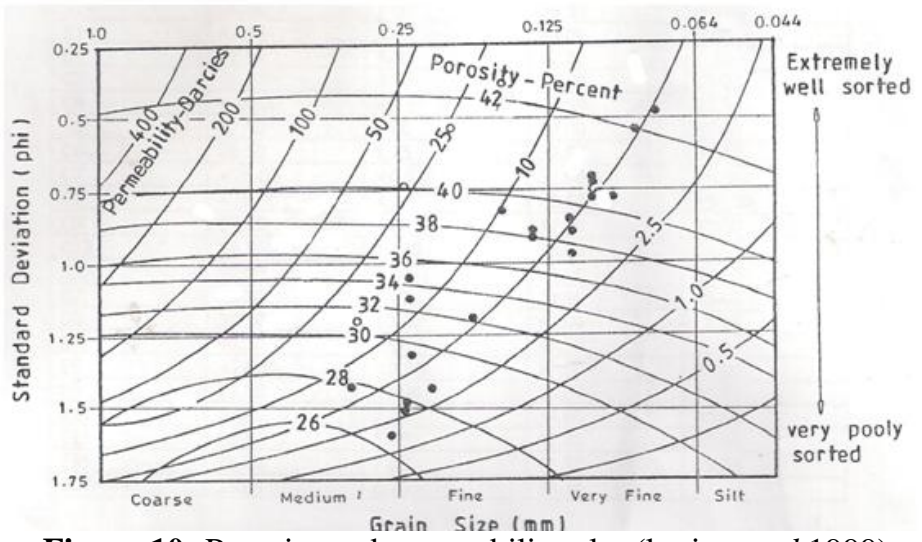

Figure 10: Porosity and permeability plot (kreisa et al 1999)

\subsection{Permeameter analysis}

Core samples of the Gombe Sandstone were subjected to falling head permeameter analysis in order to determine its porosity and permeability. The porosity evaluated ranged between $19.3 \%-25.4 \%$, with an average of $23 \%$, while the permeability ranged from $100-200 \mathrm{md}$ 'Table 3 .

Table 3 Porosity and permeability results of the Gombe Sandstone from permeameter analysis

\begin{tabular}{|c|c|c|c|c|}
\hline SAMPLE NUMBER & A9 & C7 & C18 & RCS \\
\hline Mass M(g) & 965.50 & 865.00 & 1010.10 & 1223.40 \\
\hline Length $(\mathrm{mm})$ & 60 & 50 & 60 & 76 \\
\hline Diameter $(\mathrm{mm})$ & 100 & 100 & 100 & 100 \\
\hline Volume $V=\pi r^{2} L\left(\mathrm{~cm}^{3}\right)$ & 471.24 & 392.70 & 471.24 & 596.90 \\
\hline Specific Gravity $S_{\mathrm{G}}\left(\mathrm{g} / \mathrm{cm}^{3}\right)$ & 2.65 & 2.65 & 2.65 & 2.65 \\
\hline Moisture Content W (\%) & 3.50 & 3.50 & 3.50 & 3.50 \\
\hline Test Temperature $\left({ }^{\circ} \mathrm{C}\right)$ & 27.50 & 27.50 & 27.50 & 27.50 \\
\hline Area $A=\pi D^{2} / 4\left(\mathrm{~mm}^{2}\right)$ & 7853.98 & 7853.98 & 7853.98 & 7853.98 \\
\hline Bulk Density $\rho=M / V\left(g / \mathrm{cm}^{3}\right)$ & 2.05 & 2.20 & 2.14 & 2.05 \\
\hline Dry Density $\rho_{D}=100 p /(100+e)\left(\mathrm{g} / \mathrm{cm}^{3}\right)$ & 1.98 & 2.13 & 2.07 & 1.98 \\
\hline Void Ratio e $=S_{\sigma} / \rho_{D}-1$ & 0.34 & 0.24 & 0.28 & 0.34 \\
\hline Porosity $n=(e / 1+e) * 100(\%)$ & 25.40 & 19.36 & 21.88 & 25.40 \\
\hline Standpipe diameter $\mathrm{d}(\mathrm{mm})$ & 4.50 & 4.50 & 4.50 & 4.50 \\
\hline Standpipe area a $\left(\mathrm{mm}^{2}\right)$ & 15.90 & 15.90 & 15.90 & 15.90 \\
\hline Height above outlet $\mathrm{h}(\mathrm{mm})$ & $\begin{array}{l}1460 \\
981.60 \\
660\end{array}$ & $\begin{array}{l}1460 \\
981.60 \\
660.00\end{array}$ & $\begin{array}{l}1460 \\
981.60 \\
660.00\end{array}$ & $\begin{array}{l}1460 \\
981.60 \\
660.00\end{array}$ \\
\hline Height ratios & $\begin{array}{l}1.49 \\
1.49\end{array}$ & $\begin{array}{l}1.49 \\
1.49 \\
\end{array}$ & $\begin{array}{l}1.49 \\
1.49 \\
\end{array}$ & $\begin{array}{l}1.49 \\
1.49 \\
\end{array}$ \\
\hline Test time* (min) & $\begin{array}{l}0.67 \\
0.80\end{array}$ & $\begin{array}{l}0.40 \\
0.33\end{array}$ & $\begin{array}{l}0.50 \\
0.58\end{array}$ & $\begin{array}{l}0.83 \\
0.87\end{array}$ \\
\hline Average test time $\mathrm{t}$ (min) & 0.74 & 0.37 & 0.54 & 0.85 \\
\hline $\begin{array}{l}\text { Permeability at test temp. } \\
\begin{array}{c}\mathrm{K}_{\mathrm{T}}(\mathrm{m} / \mathrm{s})=\underline{2.303 \mathrm{al}} \\
1000^{*} \mathrm{~A}^{*} 60 \mathrm{t}\end{array} * \log _{10} \frac{\mathrm{h} 1}{\mathrm{~h} 2}\end{array}$ & $1.0912 * 10^{-6}$ & $1.8186 * 10^{-6}$ & $1.0912 * 10^{-6}$ & $1.2033 * 10^{-6}$ \\
\hline $\begin{array}{l}\text { Permeability is usually given at } 20^{\circ} \mathrm{C} \\
\text { Thus: } K_{20}=K_{T}\left(\eta_{T} / \eta_{20}\right)(m / s)\end{array}$ & $9.3644 * 10^{-6}$ & $1.5607 * 10^{-6}$ & $1.2832 * 10^{-6}$ & $1.0326 * 10^{-6}$ \\
\hline Permeability K (Millidarcy) & 100 & 200 & 100 & 110 \\
\hline
\end{tabular}

\section{Discussion}

Reservoir evaluation requires data concerning individual sand bodies that are both qualitative and quantitative. The Gombe Sandstone was formed under a deltaic and braided river environment [1], and these environments generally tend to consist of thick sandstone facies [17]. Considering the laterally extensive nature and thickness of the Gombe Sandstone (over 320m) [18]. The formation can serve as a potential reservoir rock for the hydrocarbon that might have been generated in the Gongola Basin. Granulometric analysis indicated that sorting varied from well to moderate to poor'Table 1", with well to moderate sorting restricted to the thick sandstone facies of the delta front sand and channel deposits of braided river systems, while the poor is associated with the intercalated to interbedded sandstone and claystones of the delta slope environment'Figs . 5, 6, 7, 8 and 9. Skewness values are generally positive for the Gombe Sandstone, and this may suggest that, though the parking of the grains as observed from sorting is generally relatively good, but there must have been 
crystallizations of clay minerals in the voids which may have led to loss of porosity to certain extent, because positive skewness indicates occurrence of fine materials in between grains [15].Texture, amount and type of cement and interstitial clays, angularity, packing of grain and size distribution of pore spaces are the main factors affecting reservoir properties of sandstone[19],[20]. Petrographic analysis revealed that the Gombe Sandstone is generally grain supported'Figs. $11-16$.

They are usually well rounded to subangular and the clays associated with it are typically

kaolinite'Figs. 17 and 18. Authegenic and detrital kaolinite bridge pore throat and reduce porosity and permeability [21], considering this, core samples of the Gombe Sandstone were subjected to falling head permeameter test and based on this it, was observed that porosity values varied from $19-25 \%$ with average of $23 \%$, while permeability values ranged from $100 \mathrm{md}-200 \mathrm{mD}$ with average of $100 \mathrm{mD}$ (Table 3 ). In the same light porosity and permeability evaluation based on bivariate plot of [16] indicated porosity values $26-42 \%$ with an average of 35\% for the Gombe Sandstone, while permeability varied from 0.5D - 14D with an average of 6.5D"Table 1". The marked variations in the results of the two separate techniques may largely be attributed to lateral facies changes across the study location, because diagensis may have been intensive in the area where the core samples were collected compared to the other areas, because the more intense it is, the less the porosity [22].

The Gombe Sandstone when compared to reservoir rocks of major petroleum fields of prolific hydrocarbon provinces of the North Sea and Niger Delta Statfjord Formation of the North Sea displays porosity values of up to $25 \%$ with permeability in darcy ranges,[24] and the Agbada Formation of the Niger Delta has porosity ranging between $15 \%-26 \%$ with permeabilities varying between $1-2$ darcy [24]. Therefore, the Gombe Sandstone is a potential reservoir rock in the Gongola Basin of the Upper Benue Trough.

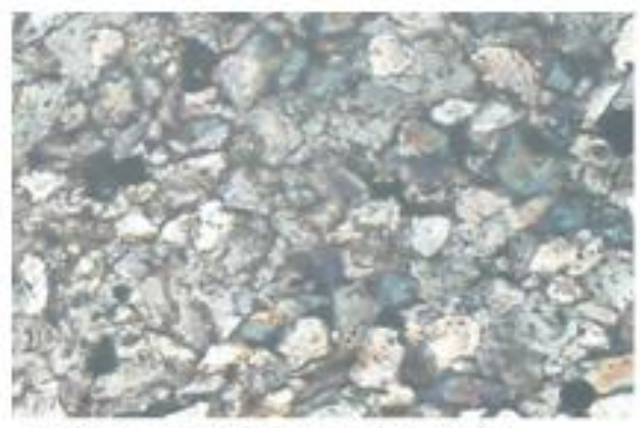

Fig. 11 well sorted grains sample $84 \times 40$

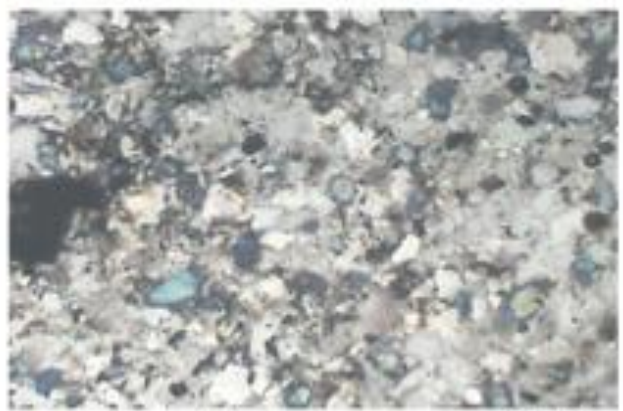

Fig. 13 Moderately sorted grains sample E5 X40

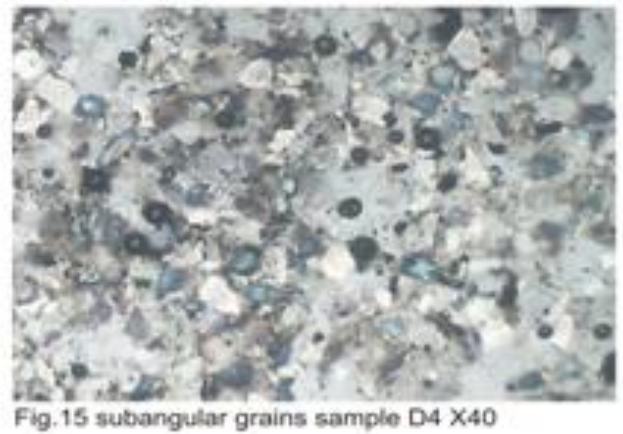

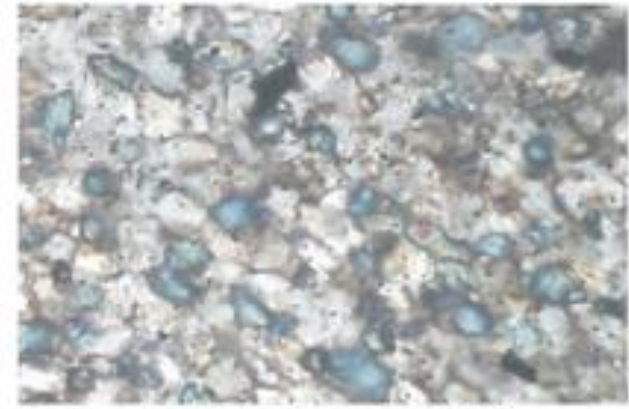

Fig. 12 well sorted grains sample D2 X40

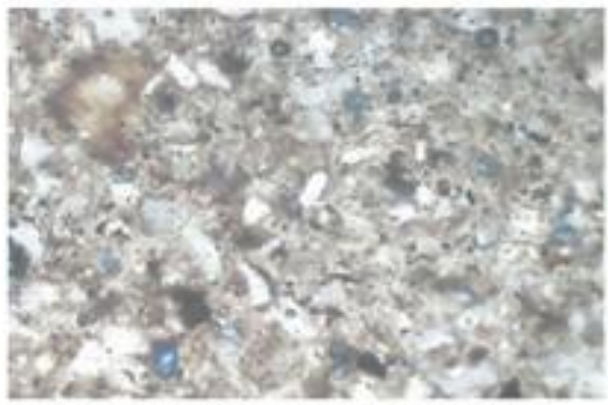

Fig.14 pocrly sorted grains sample D2 X40

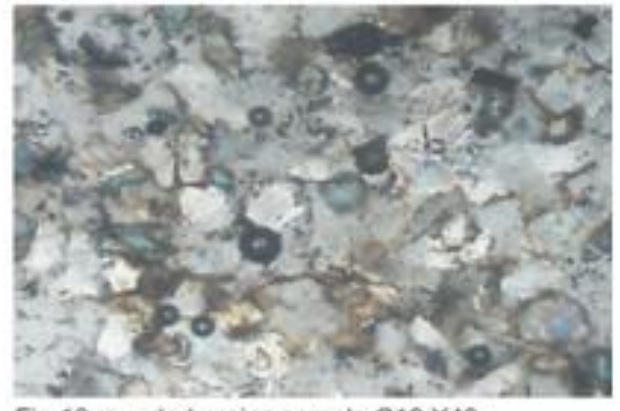

Fig-16 rounded grains sample $\mathrm{C} 18 \times 40$ 


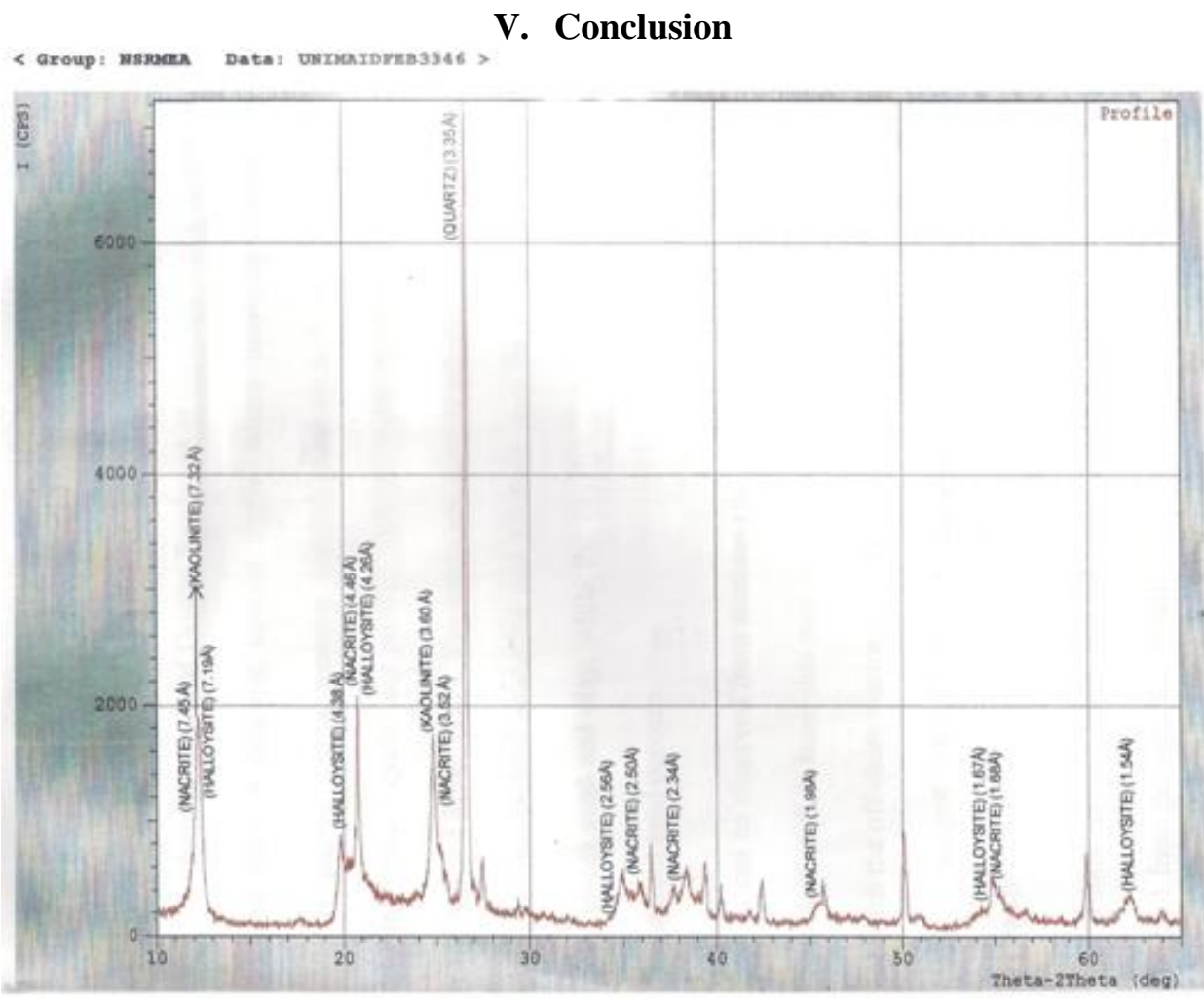

Fig.17 X-ray diffractogram of clays from Gombe Sandstone

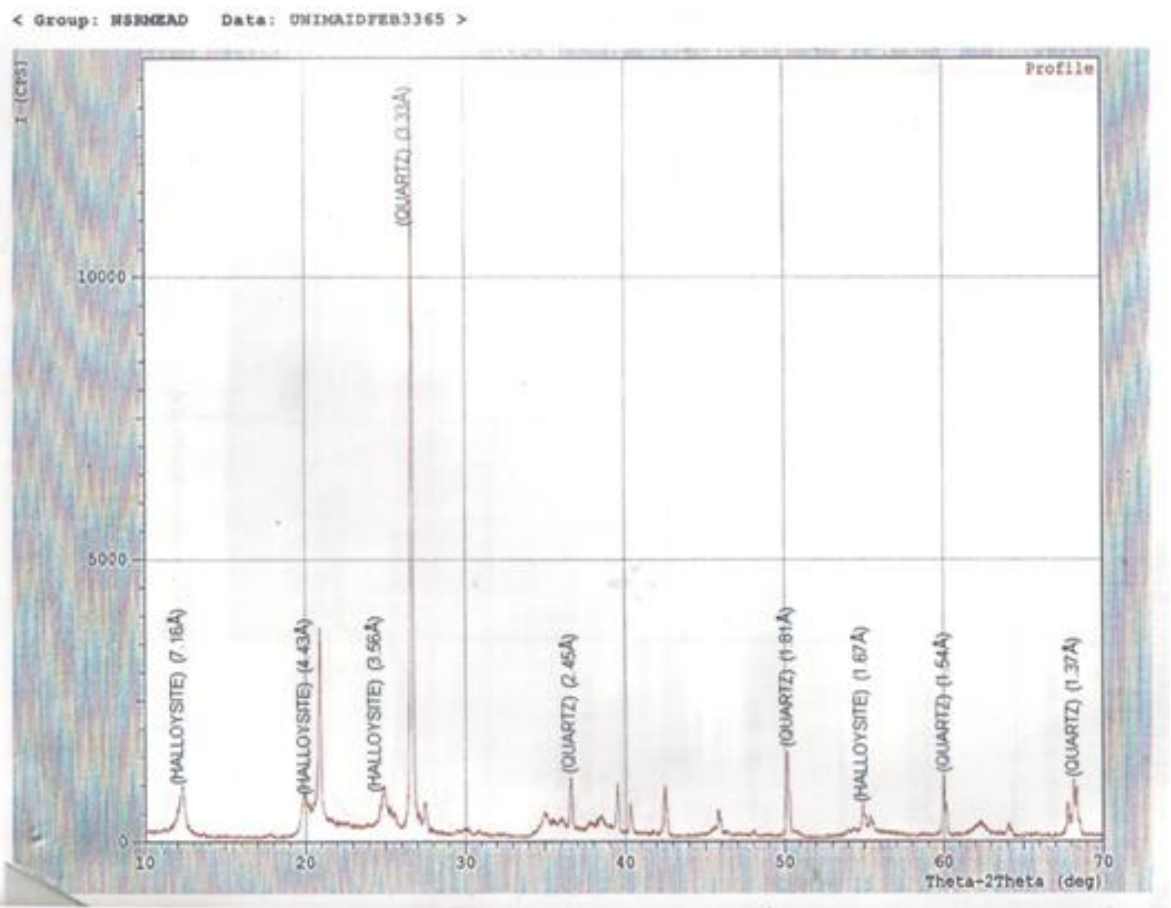

Fig.18 X-ray diffractogram of clays from Gombe Sandstone

The relative stratigraphic position of the Campano - Maastrichtian Gombe Sandstone in the overall stratigraphy of the Gongola Basin has made it a probable reservoir rock owing to the underlying source rocks of the shales of the Pindiga and Yolde Formation. Petrophysical evaluation of the Gombe Sandstones shows appreciable porosity and permeability that is comparable to that of known reservoir rocks of prolific petroleum basin of the North sea and Niger delta. Hence, the Gombe Sandstone has the ability to store and transmit hydrocarbons. 


\section{Reference}

[1]. Dike, E.F.C. and Onumara. I .S., (1999). Facies and facies architecture, and depositional environments of the Gombe Sandstone, Gombe and Environs, NE Nig. Abstr. Vol. Sci. Assoc. Nig. Annual Confer. Bauchi.

[2]. Nwajide, C.S., (2013). Geology of Nigeria's sedimentary basins. CCS Bookshop Ltd, Lagos, 86p.

[3]. Guiraud. M.,(1990). Tectono-sedimenatry framework of the Early Cretaceous continental Bima Formation (Upper Benue Trough N.E. Nigeria). Journal African Earth Sciences, 10, 341-353.

[4]. Lawal, O. and Moullade .M.,(19860. Palynological biostratigraphy of Cretaceous sediments in the Upper Benue Basin N.E. Nigeria. Revenue Micropaleotologie, 29, 61-83.

[5]. Shettima, B, (2005). Sedimentology and reconsruction of the depositional environments of the Yolde Formation in the Gongola Arm of the Upper Benue Trough (N.E. Nigeria) MSc. Thesis (unpublished), ATBU, pp.84.

[6]. Abubakar, M.B., Obaje, N.G., Lutherbacher, H.P., Dike, E.F.C. and Ashraf, A.R, (2006). A report on the occurrence of AlbianCenomanian elater-bearing pollen in the Narawa-1 well, Upper Benue Trough, Nigeria; Biostratigraphic and palaeoclimatological implication. Journal of African Earth Sciences, 45, 347-354.

[7]. Popoff. M., Wiedmann, J. and De Klazz, I., (1986). The Upper Cretaceous Gongila and Pindiga Formations, Northeeastern Nigeria. Subdivisions, age stratigraphic correlations and paleogeographic implications. Ecologea Geol. Helv., 79, 343-363.

[8]. Zaborski, P., Ugodulunwa, F., Idornigie, A., Nnabo, P. and Ibe, K.,(1997). Stratigraphy, Structure of the Cretaceous Gongola Basin, Northeastern Nigeria. Bulletin centre of Research and Production, Elf Aquitatine. 22, 153-185.

[9]. Carter, J.D., Barber, W., Tait, E.A and Jones, G.P.,(1963). The Geology of parts of Adamawa,Bauchi and Borno provinces in northeastern Nigeria. Geological Survey Nigeria Bulletin, 30:1-99.

[10]. Adegoke, O.S., Jan du Chew, R.E., Agumanu, A.E. and Ajayi, P.O., (1978). Palynology and age of the Kerri-Kerri Formation, Nigeria, Revista Espanola Micropalaco -tologia, 10, 2-283.

[11]. Dike, E.F.C. ,(1993). The Statigraphy and structure of the Kerri-Kerri Basin Northeastern Nigeria. Journal Mining and Geology, 29, 2, 77-93.

[12]. Folk, R.L., and Ward, W.C., (1957). Brazos River bar, a study in the significance of grain-size parameters. Journal of Sedimentary Petrology, 30, 514-529.

[13]. Head, K..H., (1990). Manual of soil laboratory testing, permeability, shear strength and compression tests. Oxford Uni. Press, London, 302p.

[14]. Folk, R.L., (1954). The distinction between grain size and mineral composition in Sedimentary rocks (sic) nomenclature: Jour. Geology, 62, 344-365

[15]. Folk, R.L.,(1974). Petrology of Sedimentary rocks. Austin Texas, Hemphill Book Store In: Scolle P.A. (Ed), A Colour illustrated guide to constituents, textures, cements and porosities sandstones and associated rocks.American Association of Petroleum Geologists Memoir, 28, p170.

[16]. Beard, D.C. and Weyl, D.K., (1975). Influence of texture on porosity and permeability of unconsolidated sands. American Association of Petroleum Geologists Bulletin,57, 349 -369.

[17]. Boggs, S. Jr.,( 2006). Principles of Sedimentology and Stratigraphy (4 ${ }^{\text {th }}$ eds.). Prentice Hall, Upper Saddle River, New Jersey, $1-$ $139 \mathrm{p}$.

[18]. Dessauvagie,T.F.J. (1973).Explanatory note to the geology map of Nigeria, Journal Mining and Geology 9, 3-28.

[19]. Tillman, R.W. and Martinesen, R.S., (1987). Sedimentologic model and production characteristics of Hartzo Draw field , Wyoming. Society of Sedimentary Petrologists, Tulsa Oklohoma, USA, 5-10.

[20]. Wolela, A., (2012). Diagenetic evolution and reservoir potential of the Barremian - Cenomanian Debre Libanose Sandstone, Blue Nile (Abay) Basin, Ethiopia. Journal of Cretaceous Research, 36, 83 - 95.

[21]. Pettijohn, F. J., Potter, P.E. and Siever, R. (1987), Sand and Sandstones ( $2^{\text {nd }}$ Ed). Springer- Verlag, Berlin, $407 p$.

[22]. Harris, N.B., (1989). Resevior geology of the Fangst Group (Middle Jurassic), Heidrun field, offshore mid- Norway, American Association of Petroleum Geologists Bulletin73, 1, 1415-1435.

[23]. Stanley, K.O., Jorde, K., Reastad, N., and Stockbridge, C.P., (1990). Stochostic modeling of reservoir sands bodies for inputs for reservoir stimulation, Snorre field, Northern North Sea, Norway. In: A.T. Bulller, et al.,(eds), North Sea oil and gas reservoir-II: the Norwegian institute of technology, Graham and Troman, 91-101

[24]. Harry D. and Omotola, E., (1997). Divergent/passive margin basin. In: J.D.Edwards and P.A.Santogrossi (eds), American Association of Petroleum Geologists Memoir, 46, 201-224. 